\title{
The Role of Nanotechnology in Semiconductor Industry: Review Article
}

\section{Tamirat $\mathrm{Y}^{*}$}

Department of Physics, Wolaita Sodo University College of natural and computational science, Wolaita Sodo, Ethiopia

*Corresponding author: Tamirat Y, Department of Physics, Wolaita Sodo University College of natural and computational science, Wolaita Sodo, Ethiopia, Tel: +251914555594, E-mail: my.physics3@gmail.com

Citation: Tamirat Y (2017) The Role of Nanotechnology in Semiconductor Industry: Review Article. J Mater Sci Nanotechnol 5(2): 202

Received Date: July 05, 2017 Accepted Date: August 31, 2017 Published Date: September 05, 2017

\begin{abstract}
Nanotechnology is now the key area of technology to create and manipulate materials at the nanometre (nm or $10^{-9} \mathrm{~m}$ ) $\mathrm{scale}$ either by bottom up from single groups of atoms to bulk matter or by top down which is reducing bulk materials to a group of atom. Now a days it is being used or considered for use for fabricating and constructing many efficient electronic devices which have extremely large surface area to volume ratio, this makes a large number of surface or interfacial atoms, resulting in more surface dependent material properties specially this technology is used in semiconductor industry or solar cell industry. In this article I try to review the applications of nanotechnology in semiconductor industry and more emphasis is given for Photovoltaic material application like Thin film, Quantum dot and nanowire solar cell.
\end{abstract}

Keywords: Nanotechnology; Semiconductor; Solar cell; Photovoltaic (PV)

\section{Introduction}

Nanotechnology is a field of science and technology of controlling matter on a nanoscale. It is a highly multidisciplinary field, including electrical and mechanical engineering, physics, chemistry, and biosciences. Nanotechnology will radically affect all these disciplines and their application areas [1]. It is commonly attributed for the technologies leading to produce nano-scaled materials $\left(10^{-9} \mathrm{~m}\right)$ at nanometer dimension. This feather of nano-particles provides a larger surface space per unit mass than those which are not in nano size $[2,3]$. To create nano-structured materials there are two commonly routine techniques can be used, top-down technique and bottom-up technique, which their main difference is based on the size of primary entities applied to build nano components with or without atomic level control [3-5]. One of the main applications of nanotechnology and therefore a driving force for nanoscience is the electronics industry. Over the past few decades, the transistor has been continually miniaturized. Modern integrated circuits incorporate transistors with feature as small as $32 \mathrm{~nm}$ [6]. Nanotechnology broadly includes all technologies that handle nano - scale materials, and in a narrow sense, technologies that handle unique phenomena that arise in the 10 -to-100 -nm size range. Materials of these sizes have been prepared using two techniques, the top-down and the bottom-up methods $[2,5]$.

\section{Top-Down Approach}

This approach, which leads physicists and engineers to manipulate progressively smaller pieces of matter by photolithography, Electron-beam lithography, X-ray lithography and related techniques, has operated in an outstanding way up until now [7]. It is becoming increasingly apparent, for example, that miniaturization in computer technology, which relies on silicon-based chips, is rapidly approaching the upper limits of its capabilities $[8,9]$. But it is very large on the scale of atoms and molecules. Therefore, "there is plenty of room at the bottom" for further miniaturization.

\section{Bottom-Up Approach}

An alternative and most promising strategy to exploit science and technology at the nanometer scale is offered by the bottom-up approach, which starts from nano- or subnano-scale objects (namely, atoms or molecules) to build up nanostructures. The bottomup approach is largely the realm of nanoscience and nanotechnology. This is the reason why chemists, being able to manipulate atoms and molecules, are in the ideal position to contribute to the development of nanoscience and nanotechnology [10,11].

The top-down method is applied to process just as in the semiconductor process, whereas the bottom-up method is applied to integrate molecules or atoms into nano-scale materials just as a living organism synthesizes DNA and proteins, and integrates them into a cell, and further, into a body (Figure 1) [5,12]. 


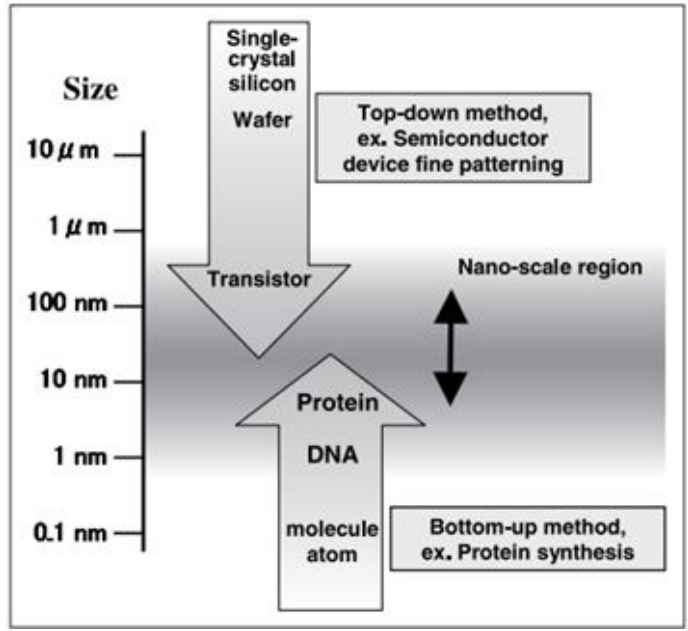

Figure 1: Two methods of approach to nano-scale [5]

The ability to fabricate structures with nanometric precision is of fundamental importance to any exploitation of nanotechnology [13]. The great promises of nanotechnology is the ability to do more in the same space: to advance our current technologies through miniaturization, so that each crop of electronics is smaller, faster, and more powerful than the one before [14].

Due to their small dimensions, nanomaterials have extremely large surface area to volume ratio, which makes a large number of surface or interfacial atoms, resulting in more "surface" dependent material properties. Nanomaterials can be classified depending on the dimensions such as (a) 0D spheres and clusters, (b) 1D nanofibers, nanowires, and nanorods, (c) 2D films, plates, and networks, (d) 3D nanomaterials as shown in Figure 2. Especially, when the sizes of nanomaterials are comparable to length, the entire material will be affected by the surface properties of nanomaterials. This in turn, may enhance or modify the properties of the bulk materials. For example, metallic nanoparticles can be used as very active catalysts. Chemical sensors from nanoparticles and nanowires enhance the sensitivity and sensor selectivity. The nanometer feature sizes of nanomaterials also have spatial confinement effect on the materials, which bring the quantum effects. The energy band structure and charge carrier density in the materials can be modified quite differently from their bulk and in turn will modify the electronic and optical properties of the materials [15].

(a)

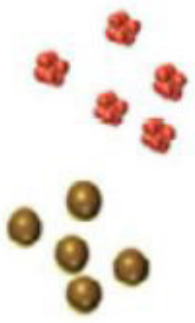

(b)

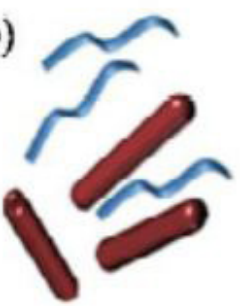

(c)

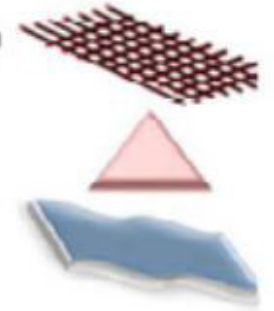

(d)



Figure 2: Classification of Nanomaterials (a) 0D spheres and clusters, (b) 1D nanofibers, wires, and rods, (c) 2D films, plates, and networks, (d) 3D nanomaterials [15]

Reduced imperfections are also an important factor in the determination of the properties of the nanomaterials. For example, the chemical stability for certain nanomaterials may be enhanced and the mechanical properties of nanomaterials will be better than the bulk materials. Nanomaterials have applications in the field of nanotechnology and displays different physical chemical characteristics from normal chemicals (i.e., silver nano, carbon nanotube, fullerene, photocatalyst, carbon nano) [15].

Carbon nanotube becomes one of the most promising materials in recently developed materials on account of its superior properties of rigidity, strength, elasticity, electric conductivity, and field emission [16]. The applications of nanotube-integrated devices include single-electron transistors, molecular diodes, memory elements and logic gates. There is great deal of interest in devising strategies to address each molecular unit individually and to interconnect them without affecting their local electronic structure adversely and also Silver nanoparticles are of interest because of the unique properties (e.g., size and shape depending optical, electrical, and magnetic properties) which can be incorporated into antimicrobial applications, biosensor materials, composite fibers, cryogenic superconducting materials, cosmetic products, and electronic components [17-22]. Several physical and chemical methods have been used for synthesizing and stabilizing silver nanoparticles [23-25].

\section{Semiconductor Quantum Dot}

Quantum dots are nanostructures in which electrons and/or holes are confined to a small region [26]. Quantum dots (QDs) as 
colloidal nanocrystalline semiconductors have unique photophysical properties due to quantum confinement effects. They emit different wavelengths over a broad range of the light spectrum from visible to infrared, depending on their sizes and chemical compositions. Compared with the traditional organic fluorophores (e.g., organic dyes and fluorescent proteins), QDs have unique optical and electronic properties, such as larger absorption coefficients, size-tunable light emission, superior signal brightness, resistance to photobleaching and simultaneous excitation of multiple fluorescence colors [27-29].

The applications of quantum dots are still mostly confined to research laboratories, but they are remarkable and often rely on the fact that quantum dots give acces to the quantum mechanical degrees of freedom of only a few carriers. Single electron transistors, the manipulation of one, or two electron spins are only some examples [30,31]. Other applications of quantum dots are related to optics: some quantum dots can confine both electrons and holes in the same region, making it possible to perform, in a solid state system, similar experiments as in atoms [32,33]. In particular, quantum dots where successfully used to produce single photons, photon pairs in an entangled polarization state and indistinguishable photons [34-36]. The combination of two or more nanostructure architectures provides another option to modulate the performance of light-harvesting devices [37,38]. The electron transport across particles is susceptible to recombination loss at the grain boundaries and charge trapping in nanostructured semiconductor films prepared from particles. The use of nanotube/nanowire support to anchor light-harvesting assemblies (e.g., semiconductor particles and dye molecules) provides a convenient way to capture photogenerated charges and transport them to electrodes. Quantum-dot-sensitized solar cells (QDSSCs) provide additional opportunities that are not available with dyesensitized solar cells (Figure 3) [39]. First, the use of quantum dots in lieu of the dye molecules provides the ability to tune the optical absorption in the solar cell through selection of semiconductor material and particle size. Second, QDSSCs can potentially exploit the recently observed multiple electron-hole pair generation per photon to achieve higher efficiencies than that predicted by Shockley and Queisser [40-43].

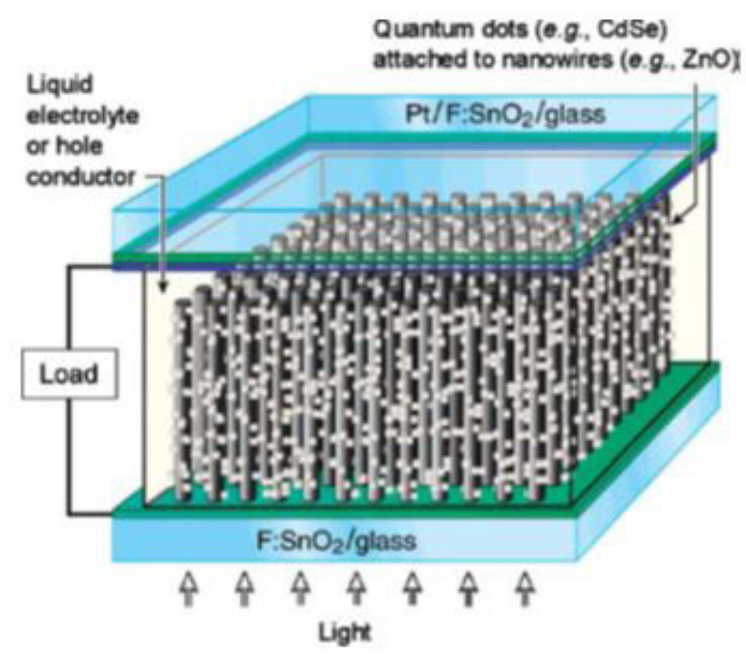

Figure 3: Schematic of a quantum-dot-sensitized solar cell (QDSSC). An array of ZnO nanowires, grown vertically from an FTO/glass substrate and decorated with CdSe quantum dots, serves as the photoanode. A second FTO/glass substrate, coated with a $100 \AA$ layer of Pt, is the photocathode. The space between the two electrodes is filled with a liquid electrolyte and the cell is illuminated from the bottom $[39,43]$

Quantum dot sensitized solar cells (QDSSCs) make use of a metal oxide electron accepting species such as $\mathrm{TiO}_{2}$. In the assembly of these devices, a layer of $\mathrm{TiO}_{2}$ is applied to a conductive material and then quantum dots are deposited. A variety of deposition methods are common, such as colloidal deposition, chemical bath deposition, SILAR (successive ionic layer adsorption and



Figure 4: Typical Schematic of a QDSSC. Light interacts with the QD, which causes electrons to be injected into $\mathrm{TiO}_{2}$ and drawn off an external circuit to generate electricity. The electrolyte then replenishes the charge carriers [52] 
reaction), and electrodeposition [44-51]. This part of the setup constitutes the QDSSCs working electrode, which is connected via an external circuit to a counter electrode. The working electrode (also called the photoanode) and counter electrode can brought close to one another with an electrolyte sandwiched in between them in the construction of an aptly named sandwich cell. The electrolyte serves to replenish the charge carriers as they are drawn off to produce a current. The overview for QDSSC is shown in Figure 4 [52].

In a bulk semiconductor electrons can freely move within an area from a few nanometers to a few hundred of nanometers as defined by the Bohr radius. Thus continuous conduction and valence energy bands exist which are separated by an energy gap. Contrary, in a quantum dot, where excitons cannot move freely, discrete atomic like states with energies that are determined by the quantum dot radius appear.

The effect of quantum confinement has a great technological interest from semiconductors and optoelectronics to biological applications. As depicted in Figure 5, by changing the particle size the emitting color of quantum dots can be tuned. Shorter quantum dots emit shorter wavelength of light and bigger quantum dots emits longer wavelengths of light. The energy band gap Eg is correlated with size: as the dimension of particles decreases, the energy increases [54].

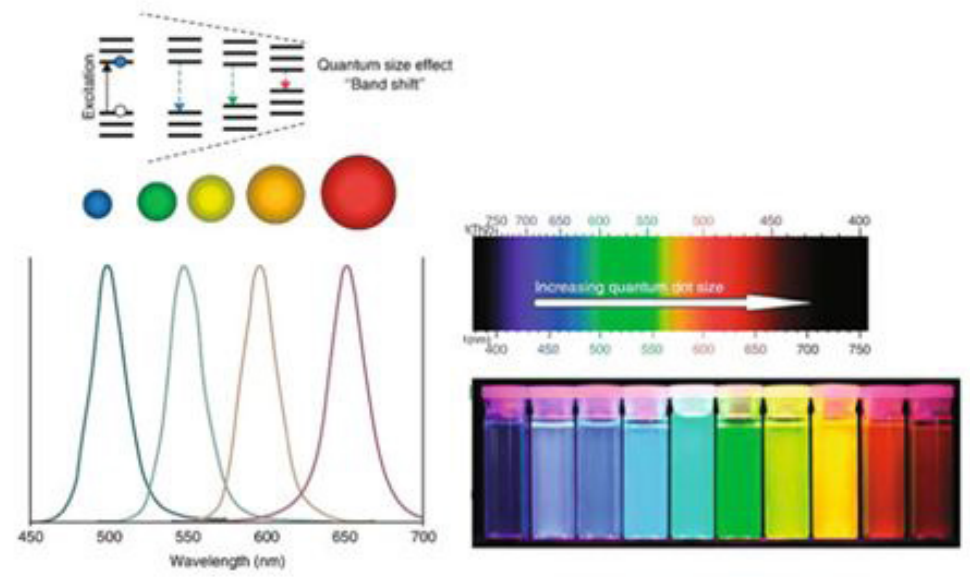

Figure 5: Schematic drawing representing the changes on optical behavior of nanoparticles associated with their size. Top: Electronic structure of QDs with "blue shift" due to quantum confinement $[53,54]$

\section{Semiconductor Nanowire}

Semiconductor nanowires (NWs) represent a unique system for exploring phenomena at the nanoscale and are also expected to play a critical role in future electronic and optoelectronic devices [55]. Over the past two decades, research in the field of semiconductor nanowires (NWs) has helped to reshape our understanding of atomic-scale crystal assembly and uncover novel physical phenomena at the nanometer scale. Compound semiconductor NWs have also been established as central components in next-generation electronic and photonic devices. This is particularly beneficial for efficient collection of photo generated carriers when core and shell segments are engineered to be thinner than minority carrier diffusion lengths. Finally, NWs are more conducive to heterogeneous integration solutions, owing to the nature of the NW geometry to accommodate heteroepitaxiallyinduced strain by relaxation along the NW free surface. This advantage effectively increases the critical thickness of latticemismatched NW crystals in comparison to epi-layers having a purely planar geometry. Therefore, NW array-based architectures are attractive for multi-junction PVs, insofar as high-efficiency devices relying on the monolithic integration of III-V materials with Si technology have been envisioned [3]. Semiconductor nanowires serve as one of the most powerful platforms available today in nanoscience given that it is now possible to design structures ab-initio and synthetically realize these structures with the structure and composition controlled from the atomic scale and up. These capabilities to design and synthetically realize complex nanowire materials are unique among nanomaterials and enable systems or building blocks to be created that have predictable physical properties [56].

\section{Semiconductor Nanowires with Novel Structure}

Semiconductor nanowires have remarkable potential for novel nanostructures and optoelectronic devices, such as heterojunctions, crystal phase quantum dots, solar cells, LED and quantum devices [57]. Graphene 2D and 1D integrated semiconductor nanostructures are exploited as a new class of nano hybrid systems that possess outstanding electronic and optoelectronic properties. In particular, fabrication of highly orientated 1D semiconductor nanowires (NWs)/nanorods (NRs) on graphene substrates can exhibit unique characteristics. The presence of graphene layer over selective substrates may support the nucleation of NWs/NRs in preferred orientations. The extraordinary properties of graphene, such as one atom thick $s p^{2}$ hybridized carbon, high carrier mobility, excellent optical transparency (97.7\%) over the visible region, flexibility and high thermal conductivity allow it to integrate it with semiconducting NWs, NRs and nanoribbons (NRbs) that may exhibit exceptional properties [58-64]. 


\section{Nanowires with High-Quality Hetero-Junctions}

The bottom-up nanowire growth mechanism allows forming hetero-junctions with high interface quality, and provides a larger freedom in combining different semiconductor materials compared to the bulk. Lattice matching is a basic requirement to form a hetero-junction without creating misfit dislocations at the interface. Because of this reason, it is quite challenging to grow heterojunctions without misfit dislocations in conventional thin film technology. However, in a nanowire, the stress at the interface between two different semiconductors is significantly smaller than that in a thin film. The reason is that a nanowire has a small footprint (usually less than $200 \mathrm{~nm}$ ) allowing elastic radial compression or expansion. This means that it is possible to stack different bandgap semiconductors with much larger lattice mismatch without creating any misfit dislocations by using nanowires. For example, a InSb/GaAs hetero-structure has been demonstrated in nanowires with an extremely large lattice mismatch of $14.6 \%$ between InSb and GaAs. In addition, a large variety of III-V materials have been axially integrated in bottom-up nanowires. This is an important advancement for material growth technology, because it may solve some long-standing technical problems that have plagued the thin film community for a long time. For instance, one of the key goals in microelectronics is to integrate optoelectronics devices made from III-V materials into traditional Si based electronic devices. In nanowire technology, this can be achieved directly by III-V nanowire growth on Si. Figure 6a,b and c show an example, where GaP and Si are epitaxially grown on top of each other within a single nanowire. Moreover, $\mathrm{GaP}$ can be vertically and epitaxially grown on a cheap substrate, e.g., $\mathrm{Si}$, as shown in Figure 6d,e and f [57].

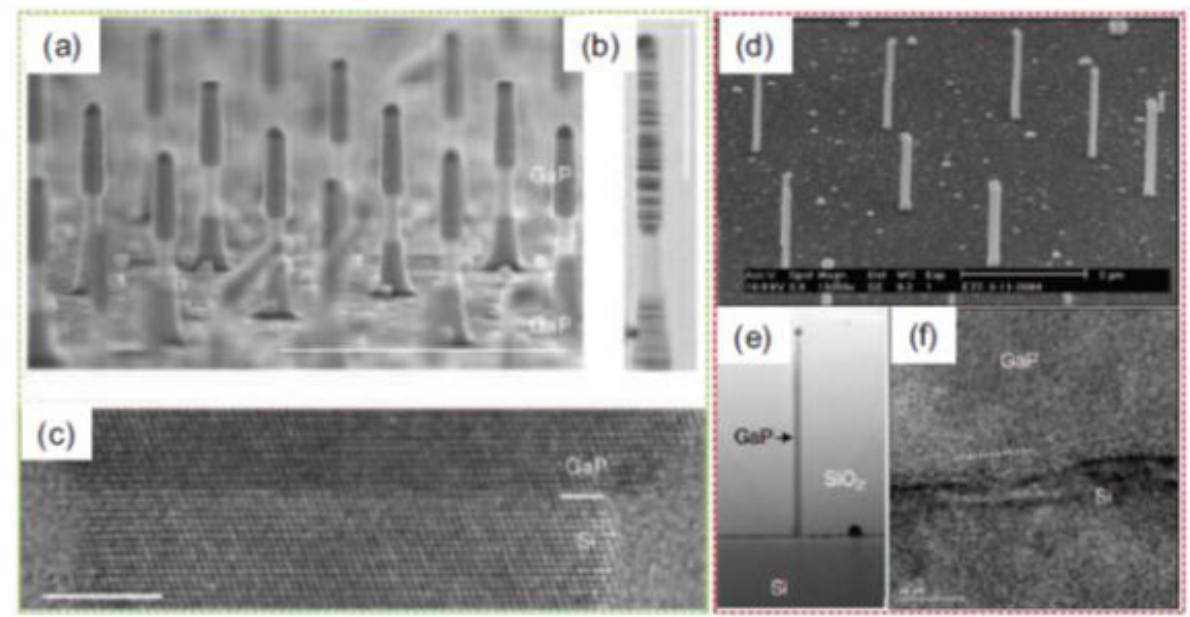

Figure 6: (a) SEM image of an array of $60 \mathrm{~nm}$ diameter $\mathrm{GaP}-\mathrm{Si}-\mathrm{GaP}$ nanowires with $\mathrm{GaP}, \mathrm{Si}$, and $\mathrm{GaP}$ segment lengths of 180,150 and $270 \mathrm{~nm}$, respectively. The sample is tilted by $80^{\circ}$, the scale bar is $1 \mu \mathrm{m}$. (b) TEM image of a single $\mathrm{GaP}-\mathrm{Si}-\mathrm{GaP}$ nanowire with a diameter of $28 \mathrm{~nm}$, scale bar is $200 \mathrm{~nm}$. (c) High-resolution TEM picture of a Si-GaP transition, scale bar is $5 \mathrm{~nm}$. (a)-(c). (d) A GaP nanowire array grown epitaxially on $\mathrm{Si}$ (111) by laser ablation. (e) Cross-sectional TEM image of a single GaP wire on Si and (f) a high-resolution TEM image of the GaP-Si interface, showing an epitaxy growth with a rotational twin dislocation at the interface [57]

\section{Technologies Based on Solar Energy}

Technologies and resources of solar energy refer to sources of energy that can be directly attributed to the light of the sun or the heat generated from the sun. In contrast, active solar energy technology refers to the harnessing of solar energy to store it or convert it for other applications and can be broadly classified into two groups: (i) Photovoltaic and (ii) solar thermal. The PV technology converts radiant energy contained in light quanta into electrical energy when light falls upon a semiconductor material by causing electron excitation and strongly enhancing conductivity. Two types of PV technology are currently available in the market: (a) crystalline silicon-based PV cells and (b) thin film technologies made from a range of different semiconducting materials, including amorphous silicon, cadmium-telluride and copper indium gallium diseline. Solar thermal technology uses solar heat, which can be used directly for either thermal or heating application or electricity generation. Accordingly, it can be divided into two categories: (i) solar thermal nonelectric and (ii) solar thermal electric. The former includes applications such as agricultural drying, solar water heaters, solar air heaters, solar cooling systems and solar cookers and the latter refers to the use of solar heat to produce steam for electricity generation, also known as concentrated solar power (CSP) [65-67]. Recent efforts to synthesize nanostructures with well-defined geometrical shapes (e.g., solid and hollow spheres, prisms, rods, tubes, and wires) and organize them as 2- and 3-dimensional assemblies have further expanded the possibility of developing new strategies for light energy conversion. Quantum dot based solar cells have drawn a lot of attention during past few years because of the possibility of boosting the energy conversion efficiency beyond the traditional Shockley and Queisser limit of 32\% for Si based solar cells [68].

Three different types of solar cells that capitalize salient properties of semiconductor nanocrystals have emerged:

(i) metal-semiconductor or Schottky junction photovoltaic cell, (ii) semiconductor nanostructure-polymer solar cell, and (iii) semiconductor sensitized quantum dot solar cell (Figure 7). Specific advantages to using semiconductor quantum dots as light 
harvesting assemblies in solar cells exist. First and foremost, their size quantization property allows one to tune the visible response and vary the band offsets to modulate the vectorial charge transfer across different sized particles. In addition, these quantum dots open up new ways to utilize hot electrons or generate multiple charge carriers with a single photon. Multiple carrier generation in $\mathrm{PbSe}$ nanocrystals has shown that two or more excitons can be generated with a single photon of energy greater than the bandgap [68-75].

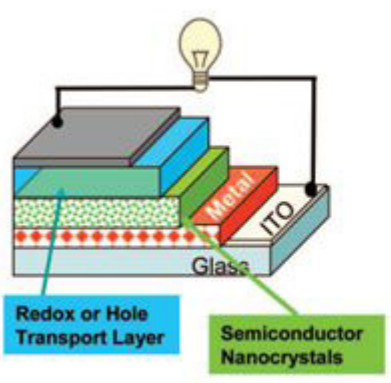

(a)

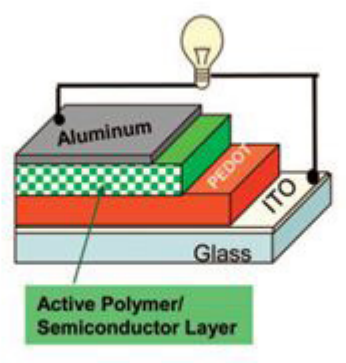

(b)



(c)

Figure 7: Schematic diagram showing the strategies to develop quantum dot (semiconductor nanocrystal) based solar cells: (a) metal-semiconductor junction, (b) polymer-semiconductor, and (c) semiconductorsemiconductor systems [75]

\section{Thin Film Application}

Thin film is a more cost-effective solution and uses a cheap support onto which the active component is applied as a thin coating. As a result much less material is required (as low as 1\% compared with wafers) and costs are decreased. Most such cells utilize amorphous silicon, which, as its name suggests, does not have a crystalline structure and consequently has a much lower efficiency (8\%), however it is much cheaper to manufacture [76-78] (Figure 8).

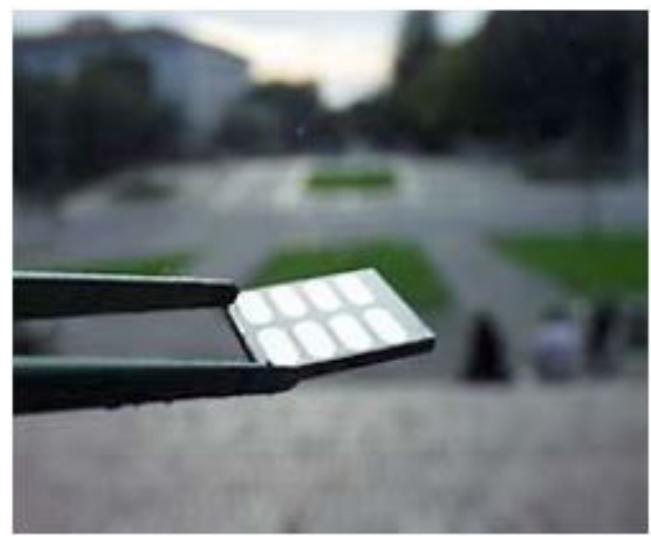

Figure 8: Picture of a solar cell, which utilizes nanorods to convert light into electricity [78,79]

To coat the nanoparticles with quantum dots tiny semiconductor crystals. Unlike conventional materials in which one photon generates just one electron, quantum dots have the potential to convert high-energy photons into multiple electrons. Quantum dots work the same way, but they produce three electrons for every photon of sunlight that hits the dots. Electrons moves from the valance band into the conduction band The dots also catch more spectrums of the sunlight waves, thus increasing conversion efficiency to as high as 65 percent. Another area in which quantum dots could be used is by making so-called a hot carrier cells. Typically the extra energy supplied by a photon is lost as heat, but with a hot carrier cells the extra energy from the photons result in higher-energy electrons which in turn leads to a higher voltage [78,80,81].

Polycrystalline thin-film solar cells such as CuInSe 2 (CIS), $\mathrm{Cu}$ (In, Ga) $\mathrm{Se}_{2}$ (CIGS), and CdTe compound semiconductors are important for terrestrial solar applications because of their high efficiency, long term stable performance and potential for low-cost production. Because of the high absorption coefficient $\left(\sim 10^{5} \mathrm{~cm}^{-1}\right)$, a thin layer of $\sim 2 \mathrm{~mm}$ is sufficient to absorb the useful part of the spectrum [67].

\section{Photovoltaic Application}

The past decade has witnessed a strong increase in the demand of clean electricity production based on renewable energy sources and an associated exponential growth of installed photovoltaic (PV) power capacity. The largest share of the actual PV electricity supply is still based on crystalline silicon wafer solar cells (SC) [82]. For photofunctional materials, nanoscale architectures usually exhibit unique optical and electronic properties. More importantly, the ability to control size and shape provides enhanced 
optoelectronic properties due to size- and shape-dependent effects and collective behaviors from the assembled building blocks. So far, numerous inorganic- and organic-based nanostructures have emerged as new building blocks to construct photocatalysis systems. However, compared to inorganic nanostructures, the organic counterparts have received special attention attributed to their considerable flexibility in molecular design, excellent tunability of the optoelectronic properties and their nice solution processability. Accordingly, the investigation of the fabrication of organic nanostructures is of great importance for photocatalytic applications [83]. One issue of particular interest is solar energy conversion using nanostructured photovoltaic devices, which can convert absorbed light into electrical energy. The use of nanowires as photovoltaic elements presents several key advantages. First, the principle of bottom-up design allows the rational control of key nanomaterial parameters, which will determine photovoltaic performance, including chemical/dopant composition, size, and morphology. Second, single or interconnected nanowire elements can be integrated with conventional electronics and/or nanoscale electronics to provide energy for low power applications. Third, single nanowire level will permit determination of the intrinsic limits, areas of improvement, potential benefits, and potentially new concepts for such nano-enabled energy conversion devices. Two unique structural motifs that can yield functional photovoltaic devices at the single nanowire level are shown schematically in Figure 9 [84-87].
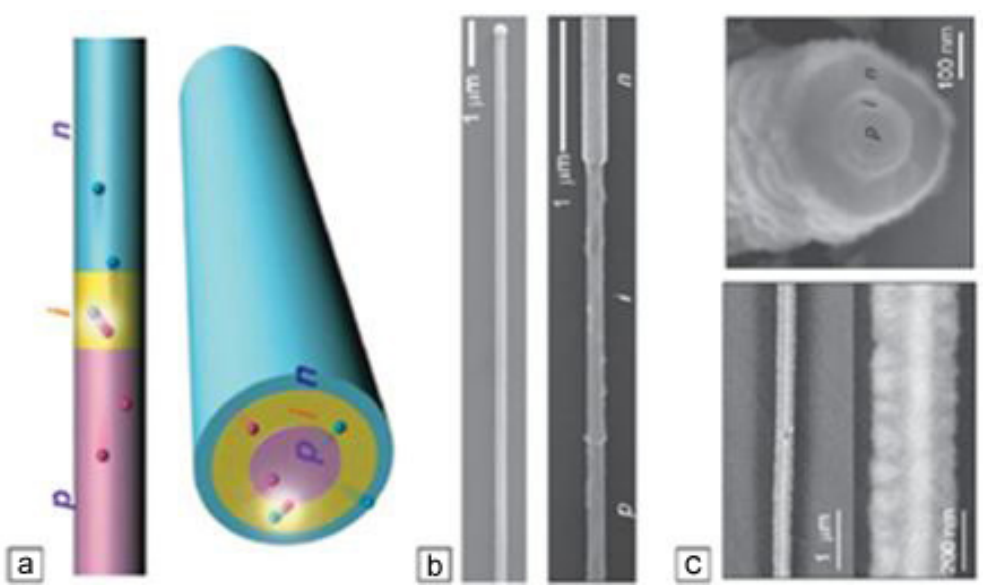

Figure 9: (a) Schematics of two distinct motifs for nanowire photovoltaics where the single p-type/intrinsic/ $\mathrm{n}$-type (p-i-n) diodes are synthetically integrated in (top) axial and (bottom) core/shell structures. (b) Scanning electron microscopy (SEM) images of $\mathrm{p}-\mathrm{i}-\mathrm{n}$ silicon nanowires. (top) As-grown nanowire with nanocluster catalyst on right tip of nanowire. (bottom) Dopantselective etched nanowire highlighting the distinct p-, i-, and n- type regions with lengths consistent with growth times. (c) SEM images of a p-i-n coaxial silicon nanowire at different magnifi cations. Images were recorded with the electron beam (left) perpendicular to the nanowire axis and (right) nearly end on [87]

\section{PV Based on Bulk Semiconductors}

PV solar cells operate by absorbing photons from incident solar radiation that have energies above the semiconductor band gap and thus create negative electrons and positive holes. Bulk inorganic semiconductors have relatively high dielectric constants, and at room temperature these photogenerated electronic particles are uncorrelated and move freely in the conduction and valence bands of the semiconductor; they are thus called free (charge) carriers. In organic semiconductors the dielectric constant is low and the photogenerated carriers are correlated and form bound electron-hole pairs that are called excitons. In the former case an internal electric field is required to efficiently separate the free electrons and holes so that they can be collected at oppositely charged electrodes and utilized in a PV cell. This electric field is most commonly produced by a p-n junction in the device; however Schottky junctions between a semiconductor and metal or liquid contacts with appropriate work function differences relative to the semiconductor material can also be used [88].

\section{Conclusion}

Nanotechnology is like a toolkit for the electronics industry. It gives us tools that allow us to make nanomaterials with special properties modified by ultra-fine particle size, crystallinity, structure or surfaces [89]. So that semiconductor is one of the materials which can be produced or fabricated by nano technology with top down or bottom up approach. Currently nano technology based semiconductor materials are becoming the basic for our modern life style due to the production of materials with extremely large surface area to volume ratio, which indicates that the electronics device we are using is small and more efficient than that we used before. Nowadays, semiconductor industry is becoming a key tool for energy harvesting with increasing its efficiency as well. One of the most impotant area that we used semiconductor is for solar energy harvesting, so that solar cell or photovolaitic materials are the one which is produced by nano technology with increasing its efficiency of photon trapping and converting to electric energy by making the material quantum dot, quantum wire and quantum well.

\section{References}

1. Ermolov V, Heino M, Karkkainen A, Lehtiniemi R, Nefedov N, et al. (2007) Significance of nanotechnology for future wireless devices and communications. IEEE $18^{\text {th }}$ Int Symposium on Personal, Indoor and Mobile Radio Communications, Athens 1-5. 
2. Kannaparthy R, Kanaparthy A (2011) The changing face of dentistry: nanotechnology. Int J Nanomedicine 6: $2799-804$.

3. Iadiz MAR, Bamedi M, Fakour SR (2017) Periodontal Diseases and Recently Applied Nano-Technology: A Review Article. Health 9: 345-51.

4. Rodgers P (2006) Nanoelecronics: Single File. Nat Nanotechnol.

5. Komatsu H, Ogasawara A (2005) Applying Nanotechnology to Electronics.16: 36-45.

6. Jan-Michael Rost (2010) Nanoscience and nanotechnology in physics and chemistry, Research Perspectives of the Max Planck Society.

7. Chaudhari A, Ibrahim M (2011) Electron Beam and X-Ray Lithography.

8. Thompson SE, Parthasarathy S (2006) Moore's law: the future of Si microelectronics. Materials Today 9: 20-5.

9. Keyes RW (2001) Fundamental limits of silicon technology. Proceedings of the IEEE 89: 227-39.

10. Balzani V (2005) Nanoscience and nanotechnology: a personal view of a chemist. Small 1: 278-83.

11. Balzani V (2008) Nanoscience and nanotechnology: The bottom-up construction of molecular devices and machines. Pure Appl Chem 80: 1631-50.

12. Semiconductor Research Corporation (2017) North Carolina, USA.

13. Emerging Applications of Radiation in Nanotechnology (2005) Proceedings of a consultants meeting held in Bologna. Int Atomic Energy Agency 1-237.

14. Wiederrecht GP (2010) Handbook of Nanofabrication $1^{\text {st }}$ Ed. Boston.

15. Sagadevan S (2013) Recent trands on nanostructures based solar energy application: a Review. Rev Adv Mater Sci 34: 44-61.

16. Lue JT (2007) Physical Properties of Nanomaterials. Encyclopedia of Nanoscience and Nanotechnology X: 1-46.

17. Rueckes T, Kim K, Joselevich E, Tseng GY, Cheung CL, et al. (2000) Carbon nanotube-based nonvolatile random access memory for molecular computing. Science 289: 94-7.

18. Bachtold A, Hadley P, Nakanishi T, Dekker C (2001) Logic circuits with carbon nanotube transistors. Science 294: 1317-20.

19. Derycke V, Martel R, Appenzeller J, Avouris PH (2001) Carbon Nanotube Inter- and Intramolecular Logic Gates. Nano Lett 1: $453-6$.

20. Hornbaker DJ, Kahng SJ, Misra S, Smith BW, Johnson AT, et al. (2002) Mapping the one-dimensional electronic States of nanotube peapod structures. Science 295: 828-31.

21. Azamian BR, Coleman KS, Davis JJ, Hanson N, Green MLH (2002) Directly observed covalent coupling of quantum dots to single-wall carbon nanotubes. Chem Commun 21:366-7.

22. Chung-Shu Wu, Chung-Yang Lee, Jem-Kun Chen, Shiao-Wei Kuo, Shih-Kang Fan, et al. (2012) Microwave-assisted Electroless Deposition of Silver Nanoparticles onto Multiwalled Carbon Nanotubes. Int J Electrochem Sci 7: 4133-42.

23. Senapati S (2005) Biosynthesis and immobilization of nanoparticles and their applications. National Chemical Laboratory, Pune, India.

24. Klaus T, Joerger R, Olsson E, Granqvist CG (1999) Silver-based crystalline nanoparticles, microbially fabricated. Proc Natl Acad Sci U S A 96: 13611-4.

25. Korbekandi H, Iravani S (2009) Silver Nanoparticles. InTech 3-37.

26. Michler P (2003) Single Quantum Dots. Springer 90: 13,18.

27. Bruchez M, Moronne M, Gin P, Weiss S, Alivisatos AP (1998) Semiconductor nanocrystals as fluorescent biological labels. Science 281: $2013-6$.

28. Ligler F, Taitt C (2002) Optical Biosensors: Present \& Future $1^{\text {st }}$ Ed. Elsevier, Chapter 17: 537-569.

29. Shao L, Yanfang Gao Y, Yan F (2011) Semiconductor Quantum Dots for Biomedicial Applications. Sensors 11: $11736-51$.

30. Likharev KK (1999) Single-Electron Devices and Their Applications. Proc IEEE 87: 606-32.

31. Koppens FH, Buizert C, Tielrooij KJ, Vink IT, Nowack KC, et al. (2006) Driven coherent oscillations of a single electron spin in a quantum dot. Nature 442: 766-71.

32. Aspect A, Dalibard J, Roger G (1982) Experimental Test of Bell's Inequalities Using Time-Varying Analyzers. Phys Rev Lett 49: $1804-7$.

33. Moehring DL, Maunz P, Olmschenk S, Younge KC, Matsukevich DN, et al. (2007) Entanglement of single-atom quantum bits at a distance. Nature 449: 68-71.

34. Michler P, Imamoglu A, Mason MD, Carson PJ, Strouse GF, et al. (2000) Quantum correlation among photons from a single quantum dot at room temperature. Nature 406: 968-70.

35. Akopian N, Lindner NH, Poem E, Berlatzky Y, Avron J, et al. (2006) Entangled photon pairs from semiconductor quantum dots. Phys Rev Lett 96: 130501.

36. Santori C, Fattal D, Vucković J, Solomon GS, Yamamoto Y (2002) Indistinguishable photons from a single-photon device. Nature $419: 594-7$.

37. Brown P, Kamat PV (2008) Quantum Dot Solar Cells. Electrophoretic Deposition of CdSe-C60 Composite Films and Capture of Photogenerated Electrons with nC60 Cluster Shell. J Am Chem Soc 130: 8890-1.

38. Sun WT, Yu Y, Pan HY, Gao XF, Chen Q, et al. (2008) CdS quantum dots sensitized $\mathrm{TiO}_{2}$ nanotube-array photoelectrodes. J Am Chem Soc 130: 1124-5.

39. Leschkies KS, Divakar R, Basu J, Enache-Pommer E, Boercker JE, et al. (2007) Photosensitization of ZnO Nanowires with CdSe Quantum Dots for Photovoltaic Devices. Nano Lett 7: 1793-8.

40. Klimov VI (2006) Detailed-balance power conversion limits of nanocrystal-quantum-dot solar cells in the presence of carrier multiplication. Appl Phys Lett 89: 123118.

41. Hanna MC, Nozik AJ (2006) Solar conversion efficiency of photovoltaic and photoelectrolysis cells with carrier multiplication absorbers J Appl Phys 100: 074510 .

42. Shockley W, Queisser HJ (1961) Detailed Balance Limit of Efficiency of p-n Junction Solar Cells. J Appl Phys 32: 510.

43. Yu K, Chen J (2009) Enhancing Solar Cell Efficiencies through 1-D Nanostructures. Nanoscale Res Lett 4: 1-10.

44. Gur I, Fromer NA, Geier ML, Alivisatos AP (2005) Air-Stable All-Inorganic Nanocrystal Solar Cells Processed from Solution. Science 310: $462-5$.

45. Chang CH, Lee YL (2007) Chemical bath deposition of CdS quantum dots onto mesoscopic TiO2 films for application in quantum-dot-sensitized solar cells. Appl Phys Lett 91: 053503.

46. Hodes G, Manassen J, Cahen D (1976) Photoelectrochemical energy conversion and storage using polycrystalline chalcogenide electrodes. Nature 261: 403-4.

47. Niitsoo O, Sarkar SK, Pejoux C, Rühle S, Cahen D, et al. (2006) Chemical bath deposited CdS/CdSe-sensitized porous TiO, solar cells. J Photochem Photobiol A Chem 181: 306-13. 
48. Baker DR, Kamat PV (2009) Photosensitization of $\mathrm{TiO}_{2}$ Nanostructures with CdS Quantum Dots: Particulate versus Tubular Support Architectures. Adv Funct Mater 19: 805-11.

49. Sun H, Mu J (2005) SILAR Deposition of CdS Thin Films on Glass Substrates Modified with 3-Mercaptopropyltrimethoxysilane. J Dispersion Sci Technol 26: 719-22.

50. Lincot D, Guillemoles JF, Taunier S, Guimard D, Sicx-Kurdi J, et al. (2004) Chalcopyrite thin film solar cells by electrodeposition. Sol Energy 77: 725-37.

51. Brown P, Kamat PV (2008) Quantum dot solar cells. Electrophoretic deposition of CdSe-C60 composite films and capture of photogenerated electrons with nC60 cluster shell. J Am Chem Soc 130: 8890-1.

52. Pernik DR (2012) Next Generation Photovoltaics: Improvements to Quantum Dot Solar Cells 1-50.

53. Mansur HS (2010) Quantum dots and nanocomposites. Wiley Interdiscip Rev Nanomed Nanobiotechnol 2: 113-29.

54. Logothetidis S (2012) Nanostructured Materials and Their Applications. Nanosci Tech, Springer.

55. Lu W, Lieber CM (2006) Topical Review: Semiconductor nanowires, J Phys D: Appl Phys 39: 387-406.

56. Naif YH (2015) A Review on Transistors in Nano Dimensions. International J Engineering Tech Sci 4: 8-18.

57. Cui Y (2015) High-efficiency nanowire solar cells. Technische Universiteit Eindhoven 1-127.

58. Park WI, Lee CH, Lee JM, Kim NJ, Yi GC (2011) Inorganic nanostructures grown on graphene layers. Nanoscale 3: $3522-33$.

59. Geim AK, Novoselov KS (2007) The rise of graphene. Nat Mater 6: 183-91.

60. R Nair, P Blake, A Grogorenko, K Novoselov, T Booth, et al. (2008) Fine Structure Constant Defines Visual Transparency of Graphene. Science 320 : 1308.

61. Lee C, Wei X, Kysar JW, Hone J (2008) Measurement of the elastic properties and intrinsic strength of monolayer graphene. Science 321: 385-8.

62. Ghosh S, Calizo I, Teweldebrhan D, Pokatilov EP, Nika DL, et al. (2008) Extremely high thermal conductivity of graphene: Prospects for thermal management applications in nanoelectronic circuits. Appl Phys Lett 92: 151911.

63. Balandin AA, Ghosh S, Bao W, Calizo I, Teweldebrhan D, et al. (2008) Superior thermal conductivity of single-layer graphene. Nano Lett 8: 902-7.

64. Biroju RK, Giri PK (2014) Controlled fabrication of graphene-ZnO nanorod, nanowire and nanoribbon hybrid nanostructures. J Nanosci Lett 4: 1-6.

65. Weiss W, Bergmann I, Stelzer R (2009) International Energy Agency Solar Heating and Cooling Program (Paris, France)

66. Kazmerski L, Gwinner D, Hicks A (2007) National Renewable Energy Laboratory, USA.

67. Sagadevan S (2013) Recent Trends on Nanostructures Based Solar Energy Applications: A Review. Rev Adv Mater Sci 34: 44-61.

68. Nozik AJ (2002) Quantum dot solar cells. Physica E 14: 115-20.

69. Kongkanand A, Tvrdy K, Takechi K, Kuno M, Kamat PV (2008) Quantum dot solar cells. Tuning photoresponse through size and shape control of CdSe-TiO2 architecture. J Am Chem Soc 130: 4007-15.

70. Ross RT, Nozik AJ (1982) Efficiency of hot-carrier solar energy converters. J Appl Phys 53: 3813-8.

71. Schaller RD, Klimov VI (2004) High efficiency carrier multiplication in PbSe nanocrystals: implications for solar energy conversion. Phys Rev Lett 92: 186601.

72. Schaller RD, Agranovich VM, Klimov VC (2005) High-efficiency carrier multiplication through direct photogeneration of multi-excitons via virtual singleexciton states. Nat Phys 1: 189-94.

73. Ellingson RJ, Beard MC, Johnson JC, Yu P, Micic OI, et al. (2005) Highly efficient multiple exciton generation in colloidal PbSe and PbS quantum dots. Nano Lett 5: 865-71.

74. Califano M, Zunger A, Franceschetti A (2004) Efficient Inverse Auger Recombination at Threshold in CdSe Nanocrystals. Nano Lett 4: 525-31.

75. Kamat PV (2008) Quantum Dot Solar Cells. Semiconductor Nanocrystals as Light Harvesters. J Phys Chem C 112: 18737-53.

76. Chopra KL, Paulson P. Dutta V (2004) Thin-Film Solar Cells: An Overview. Progress in Photovoltaics 12: 69-92.

77. Konenkamp R, Dloczik L, Ernst K, Olesch C (2002) Nano-structures for solar cells with extremely thin absorbers. Physica E 14: 219-23.

78. Nabhani N, Emami M (2013) Nanotechnology and its Applications in Solar Cells. International Conference on Mechanical and Industrial Engineering, Penang (Malaysia) 88-91.

79. Nayfeh OM, Rao S, Smith A, Therrien J, Nayfeh MH (2004) Thin film silicon nanoparticle UV photodetector. IEEE Photonics Technol Lett 16: 1927-9.

80. Catchpole KR, Polman A (2008) Plasmonic Solar Cells. Optics Express 16: 21793-800.

81. Landi BJ, Raffaelle RP, Castro SL, Bailey SG, Evans CM (2005) CdSe quantum dot-single wall carbon nanotube complexes for polymeric solar cells. Solar Energy Mat Solar Cells 87: 733-46.

82. Urs Aeberhard (2013) Simulation of nanostructure-based high-efficiency solar cells: challenges, existing approaches and future directions. IEEE J Sel Topics in Quantum Electron 19: 4000411.

83. Chen Y, Li A, Zheng-Hong H, Lu-Ning W, Kang F (2016) Porphyrin-Based Nanostructures for Photocatalytic Applications. Nanomaterials 6: 51.

84. Tian B, Zheng X, Kempa TJ, Fang Y, Yu N, et al. (2007) Coaxial silicon nanowires as solar cells and nanoelectronic power sources. Nature 449: 885-9.

85. Tian B, Kempa TJ, Lieber CM (2009) Single nanowire photovoltaics. Chem Soc Rev 38: 16-24.

86. Dong Y, Tian B, Kempa TJ, Lieber CM (2009) Coaxial group III-nitride nanowire photovoltaics. Nano Lett 9: 2183-7.

87. Lieber CM (2011) Semiconductor nanowires: A platform for nanoscience and nanotechnology. MRS Bull 36: $1052-63$.

88. Nozik AJ (2010) Nanoscience and nanostructures for photovoltaics and solar fuels. Nano Lett 10: 2735-41.

89. Rae A (2005) Real Life Applications Of Nanotechnology In Electronics. OnBoard Technology 36-9. 


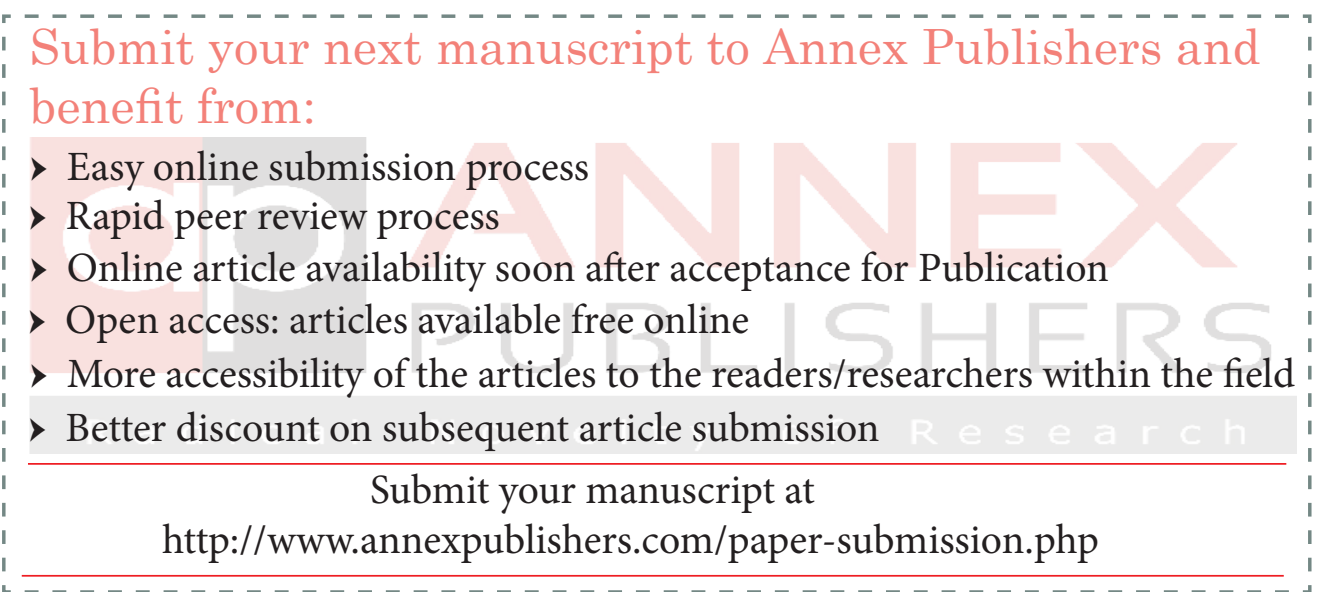

